\title{
Responding to Medical Pluralism in Practice: A Principled Ethical Approach
}

\author{
Jon C. Tilburt, MD, MPH, and Franklin G. Miller, PhD
}

The popularity of complementary and alternative medicine (CAM) raises a range of ethical issues for practicing clinicians. Principles of biomedical ethics define obligations of health care professionals, but applying principles in particular cases at the interface of CAM and biomedicine may be particularly challenging. "Recognition of medical pluralism" can help clinicians' ethical deliberations related to CAM.

Here we outline a 3-point practical approach to applying basic principles of biomedical ethics in light of medical pluralism: (1) inquiring about CAM use and the scientific evidence related to CAM, (2) acknowledging the health beliefs and practices of patients, and (3) accommodating diverse healing practices. Construed as such, recognition of medical pluralism encourages pragmatic willingness to examine the personal and cultural meaning associated with CAM use, the biases and assumptions of biomedicine, as well as the risk-benefit ratio of CAM practices. In this way, recognition of medical pluralism can help clinicians enhance patient care in a manner consistent with basic ethical principles. (J Am Board Fam Med 2007;20:489-494.)

Shared medical decision making offers a helpful framework for applying the ethical obligations of primary care clinicians articulated in the 4 principles of biomedical ethics (Table 1). ${ }^{1-4}$ However, when faced with decisions regarding complementary and alternative medicine (CAM), conventionally trained clinicians may face a range of questions that challenge their interpretation of their ethical obligations. For example, should a clinician accept a patient's request for a medical procedure based on an alternative practitioner's diagnosis? Should a clinician continue to follow a patient who does not

This article was externally peer reviewed.

Submitted 27 November 2006; revised 20 February 2007; accepted 1 March 2007.

From the Department of Clinical Bioethics (JCT, FGM) and the National Center for Complementary and Alternative Medicine (JCT), National Institutes of Health, Bethesda, MD.

Funding: none.

Prior presentation: A version of this paper was presented at the American Society for Bioethics and Humanities National Meeting in Denver, CO, October 27, 2006.

Conflict of interest: none declared.

Disclaimer: The opinions expressed are those of the authors and do not reflect the position or policy of the $\mathrm{Na}$ tional Institutes of Health, the Public Health Service, or the Department of Health and Human Services.

Corresponding author: Jon Tilburt, MD, MPH, Building 10, Room 1C 119, National Institutes of Health, Bethesda, MD 20892 (E-mail: tilburtj@cc.nih.gov). want established biomedical therapy but requests continued monitoring? Is a clinician ever justified in prescribing or referring patients to a CAM therapy that in clinical trials is demonstrated to be no better than placebo?

Approximately $36 \%$ of adults in the United States use CAM each year. ${ }^{5}$ When the definition includes "prayer for health" and "megavitamins," use rates rise to $62 \% .^{5}$ Approximately half of all CAM users in the United States seek out care from CAM practitioners. ${ }^{6}$ Recognizing the prevalence of CAM practices and the potential for adverse effects (eg, herb-drug interactions, delays in conventional care, and deceptive health claims), clinicians cannot afford to ignore CAM use in their patient populations.

In a 2005 report on $\mathrm{CAM}^{7}$ the Institute of Medicine proposed "recognition of medical pluralism" as a key value commitment to help guide health care providers in ethical deliberations related to CAM. According to the Institute of Medicine, "recognition of medical pluralism" calls for "a moral commitment to openness." Although the Institute of Medicine provided an initial formulation of this ethical concept, its relationship to accepted principles of biomedical ethics remains unclear. Here we outline a 3-point practical strategy 
Table 1. Principles of Biomedical Ethics for Clinical Practice $^{3}$

\begin{tabular}{ll}
\hline Principle & \multicolumn{1}{c}{ Clinician Obligation } \\
\hline $\begin{array}{l}\text { Respect for } \\
\text { Autonomy }\end{array}$ & $\begin{array}{r}\text { Clinicians have an obligation to respect } \\
\text { the patient as a human being who has } \\
\text { the right to make decisions consistent } \\
\text { with the patient's values. } \\
\text { Clinicians have an obligation to identify, } \\
\text { minimize or avoid harm to patients. } \\
\text { Clinicians have an obligation to seek the } \\
\text { patient's good. } \\
\text { Clinicians should promote fair } \\
\text { distribution of services for patient } \\
\text { populations. }\end{array}$ \\
\hline
\end{tabular}

of inquiry, acknowledgment, and accommodation (when warranted) for applying basic principles of biomedical ethics in a manner consistent with medical pluralism.

\section{Recognition of Medical Pluralism \\ Inquiry}

In accordance with the basic principle of nonmaleficence, clinicians should inquire about CAM use by their patients to protect them from harm. For instance, clinicians should especially inquire about a patient's use of herbal remedies such as St. John Wort (Hypericum perforatum), a botanical product used to treat mild depressive symptoms that may interact with a range of conventional medicines by induction of cytochrome P450 3A4. ${ }^{8}$ By expressing genuine interest, clinicians can elicit more accurate information about their patients' CAM use. Furthermore, consistent with the principles of nonmaleficence and beneficence, the responsibility of inquiry may call for clinicians to examine the evidence for treatments outside of their usual scope of training.

\section{Acknowledgment}

Secondly, recognition of medical pluralism encourages clinicians to acknowledge the cultural and personal meaning associated with diverse health beliefs and practices of patients. As a starting point, the health beliefs of patients deserve respectful consideration. Without necessarily endorsing all of the health beliefs and practices of patients, clinicians can recognize the legitimate role nonbiomedical approaches play in the health of the patient. Acknowledging health diversity reflects the principle of respect for patient autonomy. If patient beliefs are considered mistaken or maladaptive, patients can be counseled over time about the implications of those perspectives. ${ }^{9}$ Furthermore, such acknowledgment also may involve recognizing the limits of the biomedical paradigm as a way of explaining disease and health. ${ }^{10}$

\section{Accommodation}

Finally, in some circumstances clinicians may go beyond merely acknowledging health beliefs to actually accommodating CAM practices. When evidence demonstrates a satisfactory risk-benefit ratio for a CAM treatment, clinicians could accept or even recommend that therapy by treating it as a legitimate alternative to an established treatment. In addition, when standard treatments have failed to offer adequate benefit, medical pluralism suggests that clinicians should be prepared to assess and refer patients for seemingly safe CAM treatment even in the absence of definitive evidence of efficacy. ${ }^{11}$ Whether and to what degree to accommodate a CAM practice in a particular circumstance will depend on factors related to the stakes of the illness, the certainty of existing knowledge about treatment alternatives, the manner in which CAM will be used (as a complement versus an alternative), and the opportunity costs of pursuing CAM (eg, delays in conventional care). ${ }^{12}$

Does recognition of medical pluralism (via the strategy of inquiry, acknowledgment, and accommodation) offer any new insight into the ethical analysis of CAM in medical practice? Recognition of medical pluralism does not impose new ethical obligations. However, the approach we outline here does help in specifying the concrete meaning of ethical principles at the interface of conventional and alternative health care. As the following case analyses illustrate, the first 2 elements (inquiry and acknowledgment) specify ethical obligations relevant to every clinical interaction in which CAM may be involved. Deciding whether accommodation of a CAM practice is warranted will depend on a careful process of risk-benefit determination.

\section{Case Analyses}

Case 1

Anna, a 32-year-old woman, was told by an iridologist that she has a predisposition to colon cancer. Her family is from the Netherlands, where iridology bas been practiced in her ancestor's community for 100 years. Anna 
comes to her family physician requesting a referral for a colonoscopy. She has researched her diagnosis, received a second opinion from another iridologist, and believes she would benefit from this procedure. Anna bas no family bistory of colorectal cancer. A co-worker who gets regular colonoscopies said that the procedure is "not that bad." The physician has never heard of iridology but is skeptical about it. After conducting a complete history and physical examination, which was entirely normal, he is reluctant to refer her for an invasive procedure that he does not consider medically indicated.

Iridology is "the study of the iris, intended to identify inherited dispositions, risks and future health challenges." ${ }^{13}$ Based on iridology, the patient believes she is at increased risk of colon cancer, and she seeks out colonoscopy to mitigate her concern. Her friend's experience makes the procedure's risks seem reasonable. However, the clinician is skeptical of iridology as a diagnostic approach, thereby discounting the patient's perceived colon cancer risk. Medically speaking, the patient has an average risk profile, for which screening colonoscopy would be indicated only after age 50 . These differences in perspective illustrate how benefits and risks may be perceived and balanced differently between clinician and patient.

Recognition of medical pluralism in this case would involve the following elements. First, the clinician should refrain from prejudging the legitimacy of the patient's use of iridology. This serves 2 purposes. First, it respects the patient's autonomy. She chose to see the iridologist for some personally meaningful reason. He should inquire about the extent of her involvement, uncover the health beliefs and experiences that relate to her choice, acknowledge her right to seek out iridology, and explore her reasons for that choice. In other words, he should avoid a dismissive, unexamined opposition toward iridology merely because it is unfamiliar, implausible, or "unscientific" and instead gather complete information about her health behavior.

Secondly, avoiding a judgmental attitude in the spirit of medical pluralism will allow the clinician to undertake a helpful conversation with the patient about risks and benefits of the proposed strategy. The busy clinician might need to acknowledge his own ignorance of iridology during an initial discussion of the patient's concerns. Ideally, the clinician would gather additional reliable information on the characteristics of iridology as a screening test for colorectal cancer before a detailed discussion of possible interventions. Then recommendations could be formulated in an informed and sensitive way that recognizes the scientific uncertainty, the risks and benefits of the proposed approach, and the patient's perception of the risks. This discussion of the risks and benefits is a key step in applying nonmaleficence and beneficence in light of medical pluralism.

In this case, the risk-benefit ratio of pursuing a colonoscopy seems decidedly unfavorable. A brief search of PubMed reveals 1 study that reported a sensitivity of 0.04 for iridology as a cancer screening tool. ${ }^{14}$ The risks of colonoscopy also include possible perforation (1-2 per 1000). ${ }^{15}$

Furthermore, the costs of the procedure are substantial (ranging from \$800-\$1200 plus indirect costs). Accordingly, the principle of justice also may become relevant to ethical decision making. Is it reasonable or fair for a clinician to recommend a procedure to be paid by insurers when it is not medically indicated? Empirical evidence suggests that clinicians often include cost in decision making, especially when the potential benefits are uncertain. ${ }^{16}$ As stewards of medical resources, clinicians' concern for the justice of medical decisions is appropriate. The clinician must weigh the doubtful benefits and respect for the patient's autonomy against the personal risks and societal costs of colonoscopy. Given these considerations, the clinician should recommend against colonoscopy because the putative benefits do not outweigh the risks.

In most clinical interactions this may be the end of the decision-making process. However, viewed in light of medical pluralism, the clinician's job may not yet be done. In this case, the clinician might take an open-minded, pragmatic approach to further decision making by seeking to constructively reframe the patient's concerns. Instead of dismissing fears about cancer risk, the clinician may be warranted in recommending a less invasive cancer screening test like fecal occult blood testing (the risks and costs of which are considerably less than colonoscopy), even though iridology is medically implausible. He might also emphasize areas of colon cancer prevention where they can agree, such as improving intake of dietary fiber, reducing saturated fat, and minimizing alcohol consumption. The clinician might also educate the patient regarding warning signs that would indicate the need 
for colonoscopy for other reasons, including rectal bleeding, change in stool caliber, symptoms of anemia, etc. By refocusing discussion to areas of common agreement (which would probably mitigate her risk of colorectal cancer), the clinician can avoid making ideological disagreement the focal point of the relationship. In this manner, the principles of autonomy, nonmaleficence, beneficence, and justice, understood and balanced in light of medical pluralism, support a refusal to refer the patient for colonoscopy while maintaining a caring, proactive approach to the patient's concerns and the therapeutic relationship.

Despite sensitive conversation about the unfavorable risk-benefit ratio for colonoscopy, the patient might still decide to seek out and pay for the procedure on her own. If so, the strategy of respectful inquiry and acknowledgment, but not accommodation, would leave open the lines of communication for shared decision making in the future.

\section{Case 2}

Ruth, aged 54, was recently diagnosed with stage I breast cancer requiring lumpectomy and radiation therapy. She wishes to forego lumpectomy and radiation, instead seeking treatment from an herbalist/energy healer. She knows several women who "never felt the same" after their surgeries but has heard reports of "amazing results" from the herbalist/energy healer. Her uncle died with radiation burns from lymphoma treatment. She reasons that if the lump is so early and small, what can be the risk with "seeing how things go" with "natural" remedies? She asks her primary care doctor to follow her during her CAM treatments with periodic mammography to ensure therapy is working.

Breast cancer is an important and potentially fatal condition. Early detection and advances in therapy for stage I breast cancer have dramatically improved survival rates. CAM approaches may help manage cancer symptoms, but cannot effectively treat breast cancer. Because the stakes are high, conventional treatment is effective, and delays are potentially deadly, a clinician should have serious reservations about the patient's proposed plan on the grounds of both nonmaleficence and beneficence. At first glance, this case seems to pose a clear conflict between the ethical obligation to respect the patient's autonomy and an ethical concern for the patient's welfare.

Recognition of medical pluralism can guide this clinician's approach to shared decision making.
The pragmatic, open-minded orientation entailed by recognition of medical pluralism encourages the clinician to inquire about and acknowledge the patient's propensity to seek out CAM therapies. In adopting this approach, the clinician also might admit that biomedicine does not hold the answers for all health concerns (and at times it can expose patients to toxicity with little prospect for meaningful benefit). Respectful inquiry and acknowledgment helps to avoid conflict and animosity so that a full discussion of the risks and benefits of treatment options can proceed.

Medical pluralism also encourages the clinician to look for benefits in CAM therapies as adjuncts to standard medical treatment. In an informed and nonjudgmental manner, the clinician could then work through a range of potential treatment scenarios with both CAM and conventional treatment components based on the current literature. The clinician could propose accommodating safe CAM practices as a complementary component within a broader treatment plan that includes proven conventional care, making the patient's self-defined well-being the goal of therapy. For instance, there may be herbal strategies that would be safe adjuncts to conventional care that may improve quality of life after surgery. Observational studies of ginseng use have suggested potential benefits for breast cancer survivors' quality of life. ${ }^{17}$ In this case, the clinician may support (or at least tolerate) the patient pursuing such treatment adjuncts, preferably after healing from surgery (because there are at least theoretical bleeding risks associated with the use of ginseng). ${ }^{6}$ Such a strategy may allow the clinician to minimize the harm of delaying proven treatment (nonmaleficence) while accommodating the patient's concerns (respect for autonomy). It also would probably minimize potential for mistrust or disengagement from conventional care.

If, however, the patient persisted in opting for CAM treatment instead of conventional therapy, the clinician would be warranted in strongly voicing his objection to this course of action. In such a case, shared decision making may break down and the patient-centered orientation of the care may be strained. Although the patient retains the right to exercise her autonomy in pursuing an alternative treatment strategy despite its risks, the clinician would not be justified in endorsing such an approach. Under these kinds of circumstances, disengagement from the clinical relationship becomes a 
real possibility, either initiated by the patient or the clinician. The harms of getting no care at all from the clinician (eg, mammography and blood work) combined with the longitudinal value of their therapeutic relationship together must be balanced against the clinician's sense of professional integrity and concern about complicity with a worrisome decision. In these circumstances harm reduction assessment will be key: given that the patient has decided on this course of action, what would minimize her possibility of experiencing a bad outcome? In most circumstances, we suspect this would favor continued clinician involvement in care, just as in cases where a clinician counsels a patient engaged in other high-risk behaviors (like teens engaged in unprotected sex). Because of the benefits of some monitoring and the longitudinal value of a therapeutic relationship, respecting the differences in a single decision may be the lesser of two evils.

\section{Case 3}

Hal suffers from chronic low back pain. Initial screening tests and subsequent blood, urine, and imaging (magnetic resonance) are all normal. Conservative measures and physical therapy have provided no relief. The clinician has heard about acupuncture as a treatment for low back pain, but is not sure whether it works. The clinician searches the literature and locates 1 reasonable study testing acupuncture in low back pain: a randomized, controlled trial comparing acupuncture, sham acupuncture (ie, placebo control), and an educational bulletin. The study found that acupuncture and sham acupuncture significantly improved symptoms with no apparent side effects, but acupuncture was no better than sham acupuncture.

Because of the relative lack of conclusive studies establishing scientific benefit of many CAM practices over placebo, clinicians may be reluctant to make recommendations and referrals for CAM treatments because of an seemingly unfavorable risk-benefit ratio. Specifically, clinicians may be reluctant to refer patients for treatments that seem equivalent to placebo interventions, believing that they offer no "real" benefit. Would the clinician be ethically justified in referring the patient for acupuncture, which is equivalent to placebo treatment based on the principle of beneficence? This case raises the complex question of how determinations of benefit are made in medicine and what role the presence of medical pluralism plays in those determinations.

In modern biomedicine, therapeutic benefit is determined formally using methods of evidencebased medicine to ascertain efficacy. Typically, the standard for efficacy is superiority to a placebo control in a double-blind, randomized trial. Treatments that are no better than placebo are considered therapeutically worthless. However, recognition of medical pluralism would allow for stretching the biomedical interpretation of efficacy to include objective treatment improvements from placebo interventions themselves without violating the spirit of evidence-based medicine. Patients may derive benefit from treatments that work by virtue of a placebo effect, as evidenced by clinically meaningful superiority to a (no treatment) control group in randomized trials. ${ }^{18}$ If such superiority is demonstrated for either CAM or conventional therapies, prescribing such a therapy could be warranted provided that full disclosure is offered to the patient, there are no significant safety concerns, and no other treatment options are available. ${ }^{19}$ Such an expanded understanding of treatment benefit may support an ethically justified referral for a broader range of CAM treatments.

However, treatments that have been demonstrated to be no better than a no-treatment comparison in randomized trials would have little or no warrant for a clinician's therapeutic recommendation from an evidence-based perspective. Recognition of medical pluralism would prompt the clinician to remain both open to an expanded interpretation of efficacy and pragmatic in agreeing to reasonable and consistent evidence standards for therapeutic decisions. In the case described above, acupuncture might be a legitimate option for low back pain despite the lack of superiority to a sham acupuncture intervention.

\section{Conclusion}

Recognition of medical pluralism, as examined and applied here, helps inform the approach to challenging ethical situations in the clinician-patient relationship at the intersection of biomedicine and CAM in a manner consistent with ethical principles. It encourages clinicians to inquire about patients' use of CAM treatments, to acknowledge their diverse health concerns and beliefs, to accommodate their choice of CAM treatments when they 
do not pose undue risks, and to be willing to make referrals for CAM interventions when evidence supports a reasonable risk-benefit ratio.

The authors are grateful to Don Rosenstein, MD; Marion Danis, MD; and the anonymous manuscript reviewers for helpful suggestions on earlier versions of this paper.

\section{References}

1. Charles C, Gafni A, Whelan T. Shared decisionmaking in the medical encounter: what does it mean? Soc Sci Med 1997;44(5):681-92.

2. President's Commission for the Study of Ethical Problems in Medicine and Biomedical and Behavioral Research. Making health care decisions: the ethical and legal implications of informed consent in the patient-practitioner relationship. Washington, DC: President's Commission; 1982.

3. Beauchamp TL, Childress JF. Principles of biomedical ethics. 5th ed. Oxford: Oxford University Press; 2001.

4. Sugarman J. Informed consent, shared decisionmaking, and complementary and alternative medicine. J Law Med Ethics 2003;31(2):247-50.

5. Barnes PM, Powell-Griner E, McFann K, Nahin RL. Complementary and alternative medicine use among adults: United States, 2002. Adv Data 2004; (343):1-19.

6. Eisenberg DM, Davis RB, Ettner SL, et al. Trends in alternative medicine use in the United States, 1990-1997: results of a follow-up national survey. JAMA 1998;280(18):1569-75.

7. Institute of Medicine. Complementary and alternative medicine in the United States. Washington, DC: National Academies Press; 2005.

8. Cassileth BR, Lucarelli CD. Herb-drug interactions in oncology. New York: BC Decker; 2003.

9. Eisenberg DM. Advising patients who seek alterna- tive medical therapies. Ann Intern Med 1997;127(1): 61-9.

10. Callahan D, ed. The role of complementary and alternative medicine: accommodating pluralism. Washington, DC: Georgetown University Press; 2002.

11. Cohen MH, Eisenberg DM. Potential physician malpractice liability associated with complementary and integrative medical therapies. Ann Intern Med 2002;136(8):596-603.

12. Adams KE, Cohen MH, Eisenberg D, Jonsen AR. Ethical considerations of complementary and alternative medicine therapies in conventional medical settings. Ann Intern Med 2002;137:660-64.

13. International Iridology Practitioner's Association. Frequently asked questions. Pinehurst (TX): IIPA [cited 2006 March 1]. Available from: http://iridologyassn.org/faqs.

14. Munstedt K, El-Safadi S, Bruck F, Zygmunt M, Hackethal A, Tinneberg HR. Can iridology detect susceptibility to cancer? A prospective case-controlled study. J Alter Complement Med 2005;11(3): 515-19.

15. Gatto NM, Frucht H, Sundararajan V, Jacobson JS, Grann VR, Neugut AI. Risk of perforation after colonoscopy and sigmoidoscopy: a population-based study. J Natl Cancer Inst 2003;95(11):830-31.

16. Fetters M, Churchill LR, Danis M. Conflict resolution at the end of life. Crit Care Med 2001;29:92125.

17. Cui Y, Shu X, Gao Y, Hui C, Tao M, Zheng W. Association of ginseng use with survival and quality of life among breast cancer patients. Am J Epidemiol 2006;163(7):645-53.

18. Kaptchuk TJ. The placebo effect in alternative medicine: can performance of a healing ritual have clinical signficance? Ann Intern Med 2002;136:817-25.

19. Miller FG, Emanuel EJ, Rosenstein DL, Straus SE. Ethical issues concerning research in complementary and alternative medicine. JAMA 2004;291(5):599604. 